\title{
Superstability of generalized cauchy functional equations
}

\author{
Young-Su Lee ${ }^{1 *}$ and Soon-Yeong Chung ${ }^{2}$
}

\footnotetext{
* Correspondence: masuri@sogang. ac.kr

'Department of Mathematics, Sogang University, Seoul 121-741, Republic of Korea

Full list of author information is available at the end of the article
}

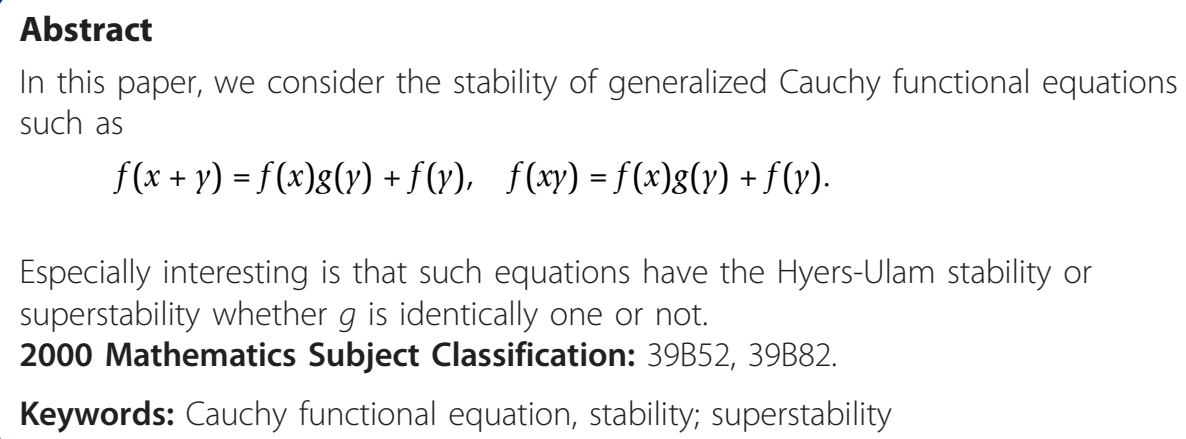

In this paper, we consider the stability of generalized Cauchy functional equations such as

$$
f(x+y)=f(x) g(y)+f(y), \quad f(x y)=f(x) g(y)+f(y) .
$$

Especially interesting is that such equations have the Hyers-Ulam stability or superstability whether $g$ is identically one or not.

2000 Mathematics Subject Classification: 39B52, 39B82.

Keywords: Cauchy functional equation, stability; superstability

\section{Introduction}

The most famous functional equations are the following Cauchy functional equations:

$$
\begin{aligned}
& f(x+y)=f(x)+f(y), \\
& f(x+y)=f(x) f(y) \\
& f(x y)=f(x)+f(y) \\
& f(x y)=f(x) f(y) .
\end{aligned}
$$

Usually, the solutions of (1.1)-(1.4) are called additive, exponential, logarithmic and multiplicative, respectively. Many authors have been interested in the general solutions and the stability problems of (1.1)-(1.4) (see [1-5]).

The stability problems of functional equations go back to 1940 when Ulam [6] proposed the following question:

Let $f$ be a mapping from a group $G_{1}$ to a metric group $G_{2}$ with metric $d(\cdot, \cdot)$ such that

$$
d(f(x y), f(x) f(y)) \leq \varepsilon .
$$

Then does there exist a group homomorphism $L: G_{1} \rightarrow G_{2}$ and $\delta_{\varepsilon}>0$ such that

$$
\begin{aligned}
& \qquad d(f(x), L(x)) \leq \delta_{\varepsilon} \\
& \text { for all } x \in G_{1} \text { ? }
\end{aligned}
$$

\section{SpringerOpen ${ }^{\circ}$}

(C) 2011 Lee and Chung; licensee Springer. This is an Open Access article distributed under the terms of the Creative Commons Attribution License (http://creativecommons.org/licenses/by/2.0), which permits unrestricted use, distribution, and reproduction in any medium, provided the original work is properly cited. 
The case of (1.1) was solved by Hyers [7]. He proved that if $f$ is a function between Banach spaces satisfying $\|f(x+y)-f(x)-f(y)\| \leq \varepsilon$ for some fixed $\varepsilon>0$, then there exists a unique additive mapping $A$ such that $\mid f(x)-A(x) \| \leq \varepsilon$. From these historical backgrounds, the functional equation

$$
E_{1}(\varphi)=E_{2}(\varphi)
$$

is said to have the Hyers-Ulam stability if for an approximate solution $\phi_{s}$ such that

$$
\left|E_{1}\left(\varphi_{s}\right)(x)-E_{2}\left(\varphi_{s}\right)(x)\right| \leq \varepsilon
$$

for some fixed constant $\varepsilon>0$ there exists a solution $\phi$ of (1.5) such that

$$
\left|\varphi_{s}(x)-\varphi(x)\right| \leq \delta_{\varepsilon}
$$

for some positive constant $\leq \delta_{\varepsilon}$.

During the last decades, Hyers-Ulam stability of various functional equations has been extensively studied by a number of authors (see [3-5,8-10]). Especially, Forti [11] proved the Hyers-Ulam stability of (1.3). The stability of (1.2) was proved by Baker, Lawrence and Zorzitto [12]. They proved that if $f$ is a function satisfying $f(x+y)-f(x)$ $f(y) \mid \leq \varepsilon$ for some fixed $\varepsilon>0$ then $f$ is either bounded or else $f(x+y)=f(x) f(y)$. In order to distinguish this phenomenon from the Hyers-Ulam stability, we call this phenomenon superstability. Generalizing results as in [12], Baker [13] proved that the superstability for (1.4) does also hold.

In this paper, we consider the stability of generalized Cauchy functional equations such as

$$
\begin{aligned}
& f(x+y)=f(x) g(y)+f(y), \\
& f(x y)=f(x) g(y)+f(y) .
\end{aligned}
$$

We say that (1.6) and (1.7) are generalized Cauchy functional equations because these are reduced the Cauchy functional equations if $g$ is identically one. It is easily checked that the general solutions of (1.6) are additive or exponential whether $g$ is identically one or not. From this point of view, we can expect that (1.6) has the HyersUlam stability or superstability due to the conditions of $g$. Actually, if $g$ is identically one in (1.6), then Hyers-Ulam stability holds [7]. On the other hand, if $g$ is not identically one in (1.6), then we shall see in Section 2 that superstability holds in this case. That is, $f$ and $g$ are either bounded or else $f(x+y)=f(x) g(y)+f(y)$.

Analogously, it is easy to see that the general solutions of (1.7) are logarithmic or multiplicative whether $g$ is identically one or not. If $g$ is identically one in (1.7), then this case is exactly the same as in [11]. And hence Hyers-Ulam stability holds in this case. We shall prove that if $g$ is not identically one in (1.7), then $f$ and $g$ are either bounded or else $f(x y)=f(x) g(y)+f(y)$.

\section{Stability of (1.6) and (1.7)}

We first consider the stability of (1.6). The general solutions of (1.6) are given by

$$
\left\{\begin{array} { l } 
{ f \equiv 0 } \\
{ g : \text { arbitrary; } }
\end{array} \quad \left\{\begin{array} { l } 
{ f : \text { constant } } \\
{ g \equiv 0 ; }
\end{array} \quad \left\{\begin{array} { l } 
{ f ( x ) = A ( x ) } \\
{ g \equiv 1 ; }
\end{array} \quad \left\{\begin{array}{l}
f(x)=a(E(x)-1) \\
g(x)=E(x),
\end{array}\right.\right.\right.\right.
$$


where $A$ is an additive mapping, $E$ is an exponential mapping and $a$ is an arbitrary nonzero constant. For the proof we refer to [[14], Lemma 1]. Although (1.6) is slightly different from (1.1), the general solutions of (1.6) are related to (1.2) rather than (1.1) if $g$ is not identically one. The stability result in the case of $g \equiv 1$ in (1.6) is well known as follows.

Theorem 2.1. [4,7]Let $E_{1}$ be a normed vector space and $E_{2}$ a Banach space. Suppose that $f: E_{1} \rightarrow E_{2}$ satisfies the inequality

$$
\|f(x+y)-f(x)-f(y)\| \leq \varepsilon
$$

for all $x, y$ in $E_{1}$, where $\varepsilon>0$ is a constant. Then the limit

$$
A(x)=\lim _{n \rightarrow \infty} \frac{f\left(2^{n} x\right)}{2^{n}}
$$

exists for all $\times$ in $E_{1}$ and $A: E_{1} \rightarrow E_{2}$ is a unique additive mapping satisfying

$$
\|f(x)-A(x)\| \leq \varepsilon
$$

for all $\times$ in $E_{1}$.

According to the above result, we know that Hyers-Ulam stability holds if $g$ is identically one. Thus, it suffices to show the case $g \otimes 1$. Especially interesting is that superstability holds if $g$ is not identically one as follows.

Theorem 2.2. Let $V$ be a vector space and let $f, g: V \rightarrow \leq$ be complex valued functions with $g \otimes 1$. Suppose that $f$ and $g$ satisfy the inequality

$$
|f(x+y)-f(x) g(y)-f(y)| \leq \varepsilon .
$$

Then, one of the following conditions holds:

(i) If $f \equiv 0$, then $g$ is arbitrary;

(ii) If $f(\mathbb{X})$ is bounded or $f(0) \neq 0$, then $g$ is also bounded;

(iii) If $f$ is unbounded, then $f(0)=0, g$ is also unbounded and $f(x+y)=f(x) g(y)+f(y)$ for all $x, y \in V$.

Proof. (i) If $f \equiv 0$, then we easily see that $g$ is arbitrary.

(ii) Suppose that $f$ is bounded and $f \otimes 0$. Then, there exists a constant $M>0$ such that $|f(x)| \leq M$ for all $x \in V$. From (2.1), it follows that

$$
|f(x) g(y)| \leq \varepsilon+2 M
$$

for all $x, y \in V$. Since $f \otimes \equiv 0$, there exists a point $x_{0}$ such that $f\left(x_{0}\right) \neq 0$. Putting $x=$ $x_{0}$ in (2.2) and dividing the result by $\left|f\left(x_{0}\right)\right|$ we have

$$
|g(\gamma)| \leq \frac{\varepsilon+2 M}{\left|f\left(x_{0}\right)\right|}
$$

for all $y \in V$. This shows that $g$ is bounded.

Now assume that $f(0) \neq 0$. Putting $x=0$ in (2.1) yields

$$
|f(0) g(\gamma)| \leq \varepsilon
$$

for all $y \in V$. We see that $g$ is bounded, since $f(0) \neq 0$. 
(iii) Finally, we are going to prove the case that $f$ is unbounded. Since $f$ is unbounded, we can take a sequence $\left\{x_{n}\right\}$ such that $\left|f\left(x_{n}\right)\right| \rightarrow \infty$. Putting $x=x_{n}$ in (2.1) and dividing both sides by $\left|f\left(x_{n}\right)\right|$ we have

$$
\left|\frac{f\left(x_{n}+y\right)}{f\left(x_{n}\right)}-g(y)-\frac{f(y)}{f\left(x_{n}\right)}\right| \leq \frac{\varepsilon}{\left|f\left(x_{n}\right)\right|} .
$$

Letting $n \rightarrow \infty$ we obtain

$$
g(\gamma)=\lim _{n \rightarrow \infty} \frac{f\left(x_{n}+\gamma\right)}{f\left(x_{n}\right)} .
$$

Substituting $x=x+x_{n}$ in (2.1) gives

$$
\left|f\left(x+x_{n}+y\right)-f\left(x+x_{n}\right) g(y)-f(y)\right| \leq \varepsilon .
$$

Dividing both sides by $\left|f\left(x_{n}\right)\right|$ and then letting $n \rightarrow \infty$ we have

$$
g(x+y)=g(x) g(y)
$$

for all $x, y \in V$. We observe that $g$ is also unbounded. If $g \equiv 0$, then from (2.1) we have

$$
|f(x+y)-f(y)| \leq \varepsilon
$$

for all $x, y \in V$. This shows that $f$ is bounded and hence this reduces a contradiction. Since $g$ satisfies (2.3) with $g \nabla 0$ and $g \otimes 1$, we conclude that $g$ is unbounded. Choose a sequence $\left\{y_{n}\right\}$ such that $\left|g\left(y_{n}\right)\right| \rightarrow \infty$. Putting $y=y_{n}$ in (2.1) and dividing both sides by $\left|g\left(y_{n}\right)\right|$ we have

$$
\left|\frac{f\left(x+y_{n}\right)}{g\left(y_{n}\right)}-f(x)-\frac{f\left(y_{n}\right)}{g\left(y_{n}\right)}\right| \leq \frac{\varepsilon}{\left|g\left(y_{n}\right)\right|} .
$$

Letting $n \rightarrow \infty$ yields

$$
f(x)=\lim _{n \rightarrow \infty} \frac{f\left(x+y_{n}\right)-f\left(y_{n}\right)}{g\left(y_{n}\right)} .
$$

We note that $f(0)=0$. Substituting $y=y+y_{n}$ in (2.1) and using (2.3) we obtain

$$
\left|f\left(x+y+y_{n}\right)-f(x) g(y) g\left(y_{n}\right)-f\left(y+y_{n}\right)\right| \leq \varepsilon .
$$

Dividing both sides in the above inequality by $\left|g\left(y_{n}\right)\right|$ and then letting $n \rightarrow \infty$ we have

$$
\begin{aligned}
f(x) g(y) & =\lim _{n \rightarrow \infty} \frac{f\left(x+y+y_{n}\right)-f\left(y+y_{n}\right)}{g\left(y_{n}\right)} \\
& =\lim _{n \rightarrow \infty} \frac{\left\{f\left(x+y+y_{n}\right)-f\left(y_{n}\right)\right\}-\left\{f\left(y+y_{n}\right)-f\left(y_{n}\right)\right\}}{g\left(y_{n}\right)} \\
& =f(x+y)-f(y) .
\end{aligned}
$$

This completes the proof. $\square$

Analogously, we are going to consider the stability of (1.7). The general solutions of (1.7) are given by 


$$
\left\{\begin{array} { l } 
{ f \equiv 0 } \\
{ g : \text { arbitrary; } }
\end{array} \quad \left\{\begin{array} { l } 
{ f : \text { constant } } \\
{ g \equiv 0 ; }
\end{array} \quad \left\{\begin{array} { l } 
{ f ( x ) = L ( x ) } \\
{ g \equiv 1 ; }
\end{array} \quad \left\{\begin{array}{l}
f(x)=b(M(x)-1) \\
g(x)=M(x)
\end{array}\right.\right.\right.\right.
$$

where $L$ is a logarithmic mapping, $M$ is a multiplicative mapping and $b$ is an arbitrary nonzero constant. In case of $g \equiv 1$, the stability result is well known as follows:

Theorem 2.3. [5,11]Let $S$ be a semigroup and $Y$ a Banach space. Further, let $f: S \rightarrow$ $Y$ be a mapping satisfying

$$
\|f(x y)-f(x)-f(y)\| \leq \varepsilon
$$

for all $x, y$ in $S$. Then the limit

$$
L(x)=\lim _{n \rightarrow \infty} \frac{f\left(x^{2^{n}}\right)}{2^{n}}
$$

exists for all $\times$ in $S$ and $L: S \rightarrow Y$ is a unique mapping satisfying

$$
\|f(x)-L(x)\| \leq \varepsilon
$$

and

$$
L\left(x^{2}\right)=2 L(x)
$$

for all $\times$ in $S$. If $S$ is commutative, then $L$ is logarithmic.

For that reason, we only consider the case $g \otimes 1$.

Theorem 2.4. Let $V$ be a vector space and let $f, g: V \rightarrow \leq$ be complex valued functions with $g \otimes 1$. Suppose that $f$ and $g$ satisfy the inequality

$$
|f(x y)-f(x) g(y)-f(y)| \leq \varepsilon .
$$

Then, one of the following conditions holds:

(i) If $f \equiv 0$, then $g$ is arbitrary;

(ii) If $f(0)$ is bounded or $f(1) \neq 0$, then $g$ is also bounded;

(iii) If $f$ is unbounded, then $f(1)=0, g$ is also unbounded and $f(x y)=f(x) g(y)+f(y)$ for all $x, y \in V$.

Proof. (i) If $f \equiv 0$, then from (2.4) we see that $g$ is arbitrary.

(ii) Suppose that $f$ is bounded and $f \otimes 0$. Then, there exists a constant $N>0$ such that $|f(x)| \leq N$ for all $x \in V$. It follows from (2.4) that we calculate

$$
|f(x) g(y)| \leq \varepsilon+2 N
$$

for all $x, y \in V$. Since $f \llbracket 0$, we see that $g$ is bounded.

Assume that $f(1) \neq 0$. Putting $x=1$ in (2.4) we have $g$ is bounded.

(iii) Now we prove the case that $f$ is unbounded. Since $f$ is unbounded, we can take a sequence $\left\{x_{n}\right\}$ such that $\left|f\left(x_{n}\right)\right| \rightarrow \infty$. Putting $x=x_{n}$ in (2.4) and dividing both sides by $\left|f\left(x_{n}\right)\right|$ we have

$$
\left|\frac{f\left(x_{n} y\right)}{f\left(x_{n}\right)}-g(y)-\frac{f(y)}{f\left(x_{n}\right)}\right| \leq \frac{\varepsilon}{\left|f\left(x_{n}\right)\right|} .
$$


Letting $n \rightarrow \infty$ we obtain

$$
g(\gamma)=\lim _{n \rightarrow \infty} \frac{f\left(x_{n} y\right)}{f\left(x_{n}\right)} .
$$

Replacing $x$ by $x x_{n}$ in (2.4) yields

$$
\left|f\left(x x_{n} y\right)-f\left(x x_{n}\right) g(y)-f(y)\right| \leq \varepsilon .
$$

Dividing both sides by $\left|f\left(x_{n}\right)\right|$ and then letting $n \rightarrow \infty$ we have

$$
g(x y)=g(x) g(y)
$$

for all $x, y \in V$. If $g \equiv 0$, then from (2.4) we have

$$
|f(x y)-f(y)| \leq \varepsilon
$$

for all $x, y \in V$. Putting $y=1$ in (2.6) we see that $f$ is bounded. This reduces a contradiction. Since $g$ satisfies (2.5) with $g \otimes 0$ and $g \otimes 1$, we can choose a sequence $\left\{y_{n}\right\}$ such that $\left|g\left(y_{n}\right)\right| \rightarrow \infty$. Putting $y=y_{n}$ in (2.4) and dividing the result by $\left|g\left(y_{n}\right)\right|$ we have

$$
\left|\frac{f\left(x+y_{n}\right)}{g\left(y_{n}\right)}-f(x)-\frac{f\left(y_{n}\right)}{g\left(y_{n}\right)}\right| \leq \varepsilon .
$$

Letting $n \rightarrow \infty$ gives

$$
f(x)=\lim _{n \rightarrow \infty} \frac{f\left(x y_{n}\right)-f\left(y_{n}\right)}{g\left(y_{n}\right)} .
$$

Putting $x=1$ yields $f(1)=0$. Replacing $y$ by $y y_{n}$ in (2.4) and using (2.5) we have

$$
\left|f\left(x y y_{n}\right)-f(x) g(y) g\left(y_{n}\right)-f\left(y+y_{n}\right)\right| \leq \varepsilon .
$$

Dividing both sides by $\left|g\left(y_{n}\right)\right|$ and letting $n \rightarrow \infty$ we obtain

$$
\begin{aligned}
f(x) g(y) & =\lim _{n \rightarrow \infty} \frac{f\left(x y y_{n}\right)-f\left(y y_{n}\right)}{g\left(y_{n}\right)} \\
& =\lim _{n \rightarrow \infty} \frac{\left\{f\left(x y y_{n}\right)-f\left(y_{n}\right)\right\}-\left\{f\left(y y_{n}\right)-f\left(y_{n}\right)\right\}}{g\left(y_{n}\right)} \\
& =f(x y)-f(y) .
\end{aligned}
$$

This completes the proof. $\square$

\section{Acknowledgements}

This work was supported by the National Research Foundation of Korea (NRF) grant funded by the Korea government (MEST) (No.2011-0000092).

Author details

${ }^{1}$ Department of Mathematics, Sogang University, Seoul 121-741, Republic of Korea ${ }^{2}$ Department of Mathematics and Program of Integrated Biotechnology, Sogang University, Seoul 121-741, Republic of Korea

\section{Authors' contributions}

$Y L$ carried out the main part of this manuscript. SC participated discussion and corrected the main theorem. All

authors read and approved the final manuscript.

\section{Competing interests}

The authors declare that they have no competing interests.

Received: 26 February 2011 Accepted: 28 July 2011 Published: 28 July 2011 


\section{References}

1. Aczél, J: Lectures on Functional Equations and Their Applications. Academic Press, New York (1966)

2. Aczél, J, Dhombres, J: Functional Equations in Several Variables. Cambridge University Press, Cambridge (1989)

3. Czerwik, S: Functional Equations and Inequalities in Several Variables. World Scientific Publishing Co., Inc., River Edge, NJ (2002)

4. Hyers, DH, Isac, G, Rassias, ThM: Stability of Functional Equations in Several Variables. Birkhäuser, Basel (1998)

5. Kannappan, PI: Functional Equations and Inequalities with Applications. Springer (2009)

6. Ulam, SM: Problems in Mordern Mathematics. Wiley, New York (1964)

7. Hyers, DH: On the stability of the linear functional equation. Proc Natl Acad Sci USA. 27, $222-224$ (1941). doi:10.1073/ pnas.27.4.222

8. Chung, J: Stability of a Jensen type logarithmic functional equation on restricted domains and its asymptotic behaviors. Adv Diff Equ 2010, 13 (2010). Art. ID 432796

9. Moghimi, MB, Najati, A, Park, C: A fixed point approach to the stability of a quadratic functional equation in $C^{*}$-algebras. Adv Diff Equ 2009, 10 (2009). Art. ID 256165

10. Rassias, ThM: On the stability of the linear mapping in Banach spaces. Proc Am Math Soc. 72, 297-300 (1978). doi:10.1090/S0002-9939-1978-0507327-1

11. Forti, GL: The stability of homomorphisms and amenability, with applications to functional equations. Abh Math Sem Univ Hamburg. 57, 215-226 (1987). doi:10.1007/BF02941612

12. Baker, J, Lawrence, J, Zorzitto, F: The stability of the equation $f(x+y)=f(x) f(y)$. Proc Am Math Soc. 74, 242-246 (1979)

13. Baker, JA: The stability of the cosine equation. Proc Am Math Soc. 80, 411-416 (1980). doi:10.1090/50002-9939-19800580995-3

14. Kannappan, PI, Sahoo, PK: On generalizations of the Pompeiu functional equation. Int J Math Math Sci. 21, 117-124 (1998). doi:10.1155/S0161171298000155

doi:10.1186/1687-1847-2011-23

Cite this article as: Lee and Chung: Superstability of generalized cauchy functional equations. Advances in Difference Equations 2011 2011:23.

\section{Submit your manuscript to a SpringerOpen ${ }^{\odot}$} journal and benefit from:

- Convenient online submission

- Rigorous peer review

- Immediate publication on acceptance

- Open access: articles freely available online

- High visibility within the field

- Retaining the copyright to your article

Submit your next manuscript at $\gg$ springeropen.com 